\title{
LA GUERRE CONSTITUANTE: RÍO DE LA PLATA, 1810-1821
}

POR

\author{
GENEVIÈVE VERDO
}

Universidad de Paris I Panthéon-Sorbona

\begin{abstract}
Inserto en la reciente historiografía revisionista del proceso independentista americano, este artículo explora sus repercusiones políticas en el Río de la Plata. La guerra contra los realistas genera en este espacio una identidad colectiva que permite explicar cómo Buenos Aires logra mantener el control politico sobre el antiguo virreinato. Aunque después del año 1820 la lucha contra los realistas sigue siendo el principal impulsor de la unión entre las provincias independientes, aparecen otro tipo de conflictos que originan una expresión peculiar del vínculo político en construcción.

Palabras clave: Guerra, independencia, Río de la Plata, construcción política, enemigo, pueblos, ejércitos.
\end{abstract}

Bien que la guerre d'indépendance constitue l'un des piliers du mythe fondateur de la nation, et que l'historiographie patriotique lui réserve une place de choix, le Río de la Plata est peut-être l'une des régions de l'Amérique espagnole les moins marquées par la guerre d'indépendance. Cette situation s'explique d'abord d'un point de vue de sa situation stratégique, le Río de la Plata présentant plusieurs particularités en regard de l'ensemble: c'est, d'une part, la seule région à avoir connu un épisode militaire important avant la crise de 1808. En résistant victorieusement aux invasions anglaises de 1806 et 1807, la cité de Buenos Aires a conquis un immense prestige, aux retombées politiques multiples. C'est, d'autre part, la seule région de l'Empire qui ne subisse pas d'invasion royaliste entre 1810 et $1820^{1}$. Il existe naturellement

1 Sur les projets de reconquête à partir de l'Espagne, Heredia, 34 (Séville, 1977): 49-63. Sur les invasions royalistes à partir du Pérou, Puente Candamo, 1980, vol. 5: 135-143. 
des tentatives de reconquête à partir du Pérou, mais celles-ci sont repoussées, tout au moins dans la partie de la vice-royauté correspondant à l'actuelle Argentine. Au contraire, Buenos Aires est dès 1810 le point de départ d'expéditions armées contre les zones loyalistes, tout d'abord le Pérou, puis le Chili. Enfin, le Río de la Plata n'est pas un espace où le conflit puisse être lu en termes de «guerre de races», étant donné la faiblesse relative de la population noire 2 (par rapport à des zones comme le Venezuela) et la faible participation des communautés indiennes au processus de mobilisation.

En dépit de ces particularités, la guerre a profondément bouleversé les pueblos du Río de la Plata, autant, si ce n'est plus, que le changement de pouvoir initié en 1810 avec la formation de la Junte de Buenos Aires. Il est même possible d'affirmer que la guerre fait intégralement partie du processus révolutionnaire, en ce qu'elle contribue à redéfinir les appartenances et les principes sur lesquels se fonde la légitimité. En ce sens, le conflit n'apparaît pas seulement comme le cadre dans lequel se déroule le processus qui mène à l'indépendance, mais bien et bien comme un événement constitutif, à plusieurs égards. A travers l'analyse de l'évolution des fronts et de la figure de l'ennemi, la guerre apparaît en effet comme l'instrument par lequel se définit la nouvelle communauté politique. De ce fait, elle influe profondément sur l'organisation de cette communauté, en imposant des choix et en faisant émerger des pratiques politiques nouvelles.

Cette approche de la guerre d'indépendance comme phénomène politique a fait l'objet, depuis quelques années, d'un important investissement de la part des historiens de l'Amérique espagnole, contribuant à renouveler en profondeur l'étude des modes de politisation dans les régions concernées. Juan Ortiz Escamilla a ainsi montré, dans le cas du Mexique, que la guerre contribuait à désarticuler l'ordre de la vice-royauté, en éloignant les pueblos des autorités royales et poussant celles-ci à reconstruire cet ordre sur d'autres bases, en confiant aux élites locales et aux communautés le soin d'organiser leur propre défense $^{3}$. Même en l'absence de révolution au sommet, la guerre contribue donc à faire émerger de nouvelles structures de gouvernement. Pour l'Amérique du sud, Anthony McFarlane a mis l'accent sur la dimension populaire de ces guerres, qui en font des vecteurs de politisation, et sur «l'importance que la guerre peut avoir sur la formation des identités politiques, des identités nationales et des institutions» ${ }^{4}$. Enfin, Clément Thibaud a prouvé dans un livre magistral à

2 Les noirs (esclaves et affranchis) représentent un peu moins d'un tiers $(27,7 \%)$ de la population totale. Bernand, 2000: 93-140.

3 Ortiz Escamilla, 1997.

4 McFarlane, 1986: 171-188. 
quel point la guerre était «un personnage de l'histoire à part entière» et, dans une large mesure, une matrice de la nation dans l'espace hispano-américain ${ }^{5}$. Dans le contexte des révolutions hispaniques, la guerre est à la fois l'agent du changement, qui provoque la fragmentation de l'espace impérial, et le miroir de la mutation révolutionnaire. Quant à l'armée, elle en vient à incarner la représentation du peuple arraché aux cadres de l'ordre corporatif. Au Venezuela et en Nouvelle-Grenade, la guerre apparaît donc, plus encore qu'ailleurs, constitutive de la politique moderne.

Jusqu'à présent, de telles analyses n'ont guère été menées pour le Río de la Plata. Le lien entre guerre et révolution, magistralement exposé par Tulio Halperín Donghi, dans une perspective essentiellement économique et sociale ${ }^{6}$, n'a pas été repris à nouveau frais dans une perspective politique, à l'exception de quelques cas isolés7. Une étude de ce genre contribue pourtant à éclaircir la problématique qui marque la période au Río de la Plata: comment Buenos Aires parvient-elle à maintenir pendant dix ans sa domination sur la plupart autres cités (au contraire de la Nouvelle-Grenade marquée par une fragmentation extrême et précoce de la souveraineté) alors même qu'elle se refuse à promulguer une Constitution? La construction par le biais de la guerre d'une «cause commune» capable de prévaloir sur les appartenances locales et de créer un lien de nature constitutionnelle (au sens d'organisation du pouvoir), semble bien être la réponse à ce mystère politique: telle est l'hypothèse qui va guider les deux premières parties de ce travail. Dans un deuxième temps, il conviendra d'étudier les retombées de la guerre dans les provinces et son rôle dans l'avènement des États provinciaux. Une fois ceux-ci constitués en 1820, la poursuite du combat pour l'indépendance constitue toujours le point nodal qui pousse les provinces à s'organiser, à chercher les voies d'une confédération, tandis que les conflits, qui changent de nature, révèle le type de souveraineté désormais à l'œuvre.

\section{LA GUERRE COMME VECTEUR DE L'APPARTENANCE}

Il s'agit tout d'abord de présenter l'évolution des différents fronts, dans un espace qui se présente dès le début du conflit comme un foyer de «libération» - entendons par là la diffusion, par le biais des armes, de l'idéologie patriotique et réformiste qui caractérise la Révolution de Mai. Cette évolution fait

\footnotetext{
5 Thibaud, 2003.

6 Halperín Donghi, 1972; 1978: 121-158.

7 Tio Vallejo, 2002: 355-388. Meisel, 2002: 439-451.
} 
apparaître des ennemis de nature différente, qui permettent de nuancer la qualification de ce conflit comme simple «guerre d'émancipation». Des positions stratégiques aux qualifications discursives, on voit se dessiner les contours d'une communauté d'appartenance, dont les frontières, tant géographiques que symboliques, correspondent à la nation que les révolutionnaires s'efforcent de construire.

Dès sa formation, en mai 1810, la Junte s'emploie à mettre sur pied une armée composée des bataillons de milice de la capitale, pompeusement intitulée «Expédition d'Aide aux provinces intérieures» (Expedición Auxiliar a las Provincias Interiores $)^{8}$. Les troupes qui quittent la capitale en juin 1810 sont accompagnées de commissaires politiques, chargés d'obtenir l'adhésion des autres cités et d'y organiser des élections. La plupart des cités s'étant déjà ralliées à Buenos Aires, l'expédition ne rencontrera de véritable résistance qu'à Córdoba, dont le gouverneur, fidèle au vice-roi du Pérou et à la Régence, sera assassiné en août 1810 avec ses principaux partisans ${ }^{9}$. À partir de Córdoba, le corps expéditionnaire se scinde en deux. Une partie remonte vers le Paraguay, sous la direction de Manuel Belgrano, tandis que l'autre poursuit sa route vers le Haut Pérou, sous la direction de Juan José Castelli. Le premier front ouvert sur les marges de la vice-royauté est donc celui du Paraguay, où les porteños affrontent sans succès d'autres créoles patriotes. Toutefois, les défaites répétées de Belgrano se soldent par la conclusion d'un armistice en mars 1811, à partir duquel la frange nord du Río de la Plata fait sécession et acquiert son autonomie ${ }^{10}$.

Le deuxième front, plus important, se prolonge jusqu'en 1825: c'est celui du Haut Pérou, où les troupes de Buenos Aires rencontrent les forces loyalistes envoyées depuis Lima par le vice-roi Abascal. Durant quinze ans, ce front avance et recule au gré des offensives et des contre-offensives des deux armées rivales, auxquelles il faut ajouter les guerrillas patriotes du Haut Pérou ${ }^{11}$. Du côté du Río de la Plata, c'est l'Armée du Nord, issue de l'Expédition d'Aide, qui occupe ce front jusqu'en 1815. Bien qu'elle soit composée de bataillons et de milices de différentes cités, on peut la considérer comme une armée régulière, dans la mesure où elle est dirigée par des généraux prestigieux (Belgrano, Rondeau) nommés par le pouvoir central à Buenos Aires. Durant cette période, l'armée est cantonnée dans les provinces de Salta et de Tucumán, qu'elle marque fortement de son empreinte. Après 1815 et la grande dé-

\footnotetext{
8 Bidondo, 1987; 37 (Buenos Aires, 1988): 337-375.

9 García Belsunce, 1961, vol. 6: 153-175.

10 Díaz Bellido, 1999.

11 Demélas-Bohy, 2007.
} 
faite de Sipe Sipe, la défense de la zone est confiée au gouverneur de la province de Salta, Martín Güemes, qui conduit sa propre armée de gauchos. Il s'agit d'une une armée provinciale, plus proche de la guerrilla en termes d'organisation, qui parvient à tenir le front et à repousser huit invasions royalistes jusqu'à la mort de son chef, en $1821^{12}$. Dans la même logique de lutte contre les royalistes, qui ont reconquis le Chili en 1814, un troisième front est ouvert par San Martín avec sa fameuse traversée des Andes, en 1817. San Martín est à la tête d'une armée entièrement forgée par ses soins, composée essentiellement d'éléments provinciaux, auxquels se joignent ensuite les patriotes chiliens.

Le dernier front, d'une tout autre nature, est celui de la Bande Orientale. Sa configuration est plus complexe, car plusieurs ennemis s'y succèdent et s'y entrecroisent. Ce sont tout d'abord les loyalistes, menés par le vice-roi Elío, dont la lutte s'inscrit dans une logique d'affrontement entre Montevideo, qui a reconnu le Conseil de Régence, et Buenos Aires. Cette campagne, qui dure de 1810 à 1814, est marquée par des épisodes célèbres telle la victoire de San Lorenzo, remportée par San Martín en 1813, ou le blocus de Montevideo dans lequel s'illustrent Rondeau et Alvear. En second lieu se trouvent les Portugais, qui interviennent aux côtés des loyalistes en 1811, puis envahissent une nouvelle fois la Bande Orientale en 1816. Cette seconde invasion occasionne des négociations incessantes avec les dirigeants de Buenos Aires de 1816 à $1819^{13}$.

Le troisième ennemi que doivent affronter les troupes de Buenos Aires est le caudillo de la Bande Orientale, José Gervasio Artigas. Au début du conflit, ce capitaine d'un corps de milices de la campagne, les Bladengues, offre ses services aux généraux de Buenos Aires pour lutter contre les loyalistes. L'alliance perdure jusqu'en 1812, moment où Artigas, soucieux de préserver son autonomie, refuse d'intégrer son armée à celle de Buenos Aires. En février 1813, il est déclaré traitre à la patrie et on invite à la défection dans son camp; en avril de la même année, les députés qu'il envoie à l'Assemblée Générale Constituante ne sont pas acceptés. Désormais, Artigas fait cavalier seul et combat à la fois les royalistes et les porteños. A partir de 1814-1815, il parvient à prendre le contrôle de la région du Littoral (provinces de Santa Fé, Entre Rios et Córdoba), et fonde la «Ligue des Peuples Libres», opposée au centralisme de Buenos Aires ${ }^{14}$.

12 Cornejo, 1946. Frías, 1971-1973. Figueroa Güemes, 1971. Academia Nacional de la Historia, 1973: 67-77.

13 Salas, XV/2 (Berlin, 1989): 193-231; Segreti, 1994.

14 Sur le rôle d'Artigas dans la révolution, Acevedo, 1950. Caillet-Bois, 46 (Buenos Aires, 1973): 111-124. Reyes Abadie, 1975. Azcuy Ameghino, 1986. Petit Muñoz, 1988. 
Ce dernier conflit, qui dégénère de plus en plus gravement, sera fatal à la révolution porteña. En effet, ce sont les attaques des caudillos du Littoral, alliés d'Artigas, qui finissent par avoir raison du régime instauré par Buenos Aires en 1810. La bataille de Cepeda, en février 1820, sonne le glas du Directoire et précipite l'indépendance des différentes provinces.

L'évolution de ces différents fronts s'accompagne d'une transformation dans la figure de l'ennemi, sans cesse dénoncé dans les sources, et qui reflète $a$ contrario la communauté politique que l'on s'efforce de promouvoir. L'ennemi désigné par le nouveau régime apparaît tout d'abord sous les traits des mandones, les agents de la Couronne qui forment l'administration de la vice-royauté. La défiance et la vindicte que les créoles patriotes manifestent à leur égard se cristallise en partie sur leur origine géographique, la plupart provenant de la péninsule, mais surtout sur le fait qu'ils soient des partisans de la Régence, donc d'un certain conservatisme, face au désir de réforme des patriotes. Il s'agit donc d'une opposition essentiellement idéologique, qui vient se greffer sur le ressentiment traditionnel que nourrissent les créoles vis-à-vis des péninsulaires pourvus de charges officielles ${ }^{15}$.

Dans le discours, ces mandones sont décrits comme des praticiens de l'arbitraire, de la violence, poursuivant des fins personnelles. L'accusation lancée contre Juan Gutiérrez de la Concha, gouverneur de Córdoba, accusé de «[confondre] les droits du roi et les usurpations de sa propre personne» et déclaré «ennemi public de l'État» ${ }^{16}$, apparaît comme un paradigme dessinant les traits de l'ennemi. Ces caractéristiques s'appliquent également au vice-roi du Pérou, Abascal, sous les ordres duquel agit le gouverneur. Champion de l'absolutisme et de la conservation du statu quo, Abascal a en effet réuni sous sa tutelle les provinces du Haut Pérou et de Córdoba en juillet 1810, dans le but de «repousser toute tentative hostile de la part de la Junte de gouvernement de Buenos Aires» ${ }^{17}$. À l'inverse, les membres de la Junte, dans leurs textes de propagande, se présentent comme animés d'un esprit de tolérance, praticiens de la mesure, de la douceur, soucieux d'assurer le bonheur public. Nonobstant la sévérité dont usent les commissaires de la Junte envers leurs ennemis, les rapports entre le nouveau pouvoir et les cités sont décrits sous l'angle de la fraternité, de la justice et de l'esprit de réforme. Il s'esquisse donc à travers ces redéfinitions une double évolution: d'une part le pouvoir des fonctionnaires royaux est peu à peu discrédité au profit de la souveraineté des pueblos; d'autre part l'esprit de réforme qui anime la Junte, porte-parole des pueblos,

\footnotetext{
15 Guerra, 1992: 73-84.

16 Circular de la Junta á los Cabildos, 27 de junio de 1810, in Carranza, 1894, vol. 1: 7.

17 Puente Candamo, 1980, vol. 5: 135-143.
} 
prend le pas sur le conservatisme égoïste des mandones. Par cette première campagne de dénonciation, qui a lieu dans les tout premiers mois de la révolution, la légitimité change de camp, sous les auspices de la conservation des droits de Ferdinand VII.

Après la destitution des fonctionnaires royaux, la définition de l'ennemi s'étend à tous les Espagnols «européens» officiellement persécutés à partir de 1812 , et contraints de se faire naturaliser ${ }^{18}$. En moins de deux ans, l'opposition idéologique des débuts a pris le sens d'un affrontement entre deux communautés «nationales», les Espagnols européens étant tous soupçonnés d'être des ennemis de la révolution. Ce discours radical, forgé à la faveur des combats, se retrouve dans toute l'Amérique espagnole. Il s'agit de diaboliser l'ennemi, de discréditer les conquérants d'hier pour mieux justifier l'entreprise des patriotes. Confondu avec le despotisme, qu'il incarne, l'«Espagnol» se voit chargé de tous les maux de la Conquête et des qualificatifs qui désignent le pouvoir arbitraire: tour à tour qualifié de «barbare», «cruel», «sanguinaire», «despote», il est rejeté au ban de l'humanité. On voit clairement à l'œuvre dans cet anathème la construction idéologique qui préside à la définition d'une nouvelle communauté, rendue effective en 1813 par la création d'une «nationalité» propre au Río de la Plata ${ }^{19}$. Il s'agit bien ici de dessiner les contours d'un nouveau corps politique, selon le sens que revêt le mot «nation» à l'époque révolutionnaire ${ }^{20}$. En ce sens, parce qu'ils sont rejetés hors du corps de la nation, les Espagnols européens peuvent être qualifiés d'ennemis «extérieurs». Bien qu'il s'agisse d'une guerre civile, la logique suivie est de plus en plus celle d'une guerre d'indépendance: «nationaliser» le conflit revient à extérioriser l'ennemi, et vice-versa. Toutefois, à la même époque, le thème de l'ennemi «intérieur» fait également son apparition. Ce terme, employé par les acteurs eux-mêmes, désigne le patriote dénaturé, le membre de la communauté qui agit contre le bien de celle-ci, principalement en se retranchant de l'action ou en poursuivant des fins personnelles contraires au bien commun. Les épithètes qui désignent le plus souvent ces personnages sont ceux d'égoïstes, de rebelles, de factieux: leur plus grand crime est de favoriser la dissolution de l'ordre social, à l'encontre de la communauté que l'on s'efforce de construire.

Le personnage sert d'incarnation à ce modèle est Artigas, livré à la vindicte des patriotes porteños dès 1812. Cette opposition acharnée explique notam-

18 Decreto de la Soberana Asamblea General, 6 de febrero de 1813, in Sampay, 1975: 135.

19 Vogel, LXXI/1 (Durham, 1991): 107-131.

20 Hobsbawm, 1992. De fait, dans les décrets, les termes de «nationalité» et de «citoyenneté» sont sans cesse confondus, la naturalisation entraînant automatiquement la concession des droits politiques. 
ment le refus obstiné des députés de 1813 d'envisager le fédéralisme, promu par le caudillo de la Bande Orientale, comme solution politique à l'union des provinces du Río de la Plata ${ }^{21}$. Elle justifie également la condamnation d'une certaine forme de démocratie directe, pratiquée par le chef des Orientaux du fait de son statut de chef de guerre. Cette définition de l'ennemi intérieur sert donc de marqueur politique au même titre que l'opposition aux Espagnols. Ces deux figures de l'ennemi ne vont d'ailleurs cesser de coexister, la seconde devenant de plus en plus présente dans le discours du fait de la menace croissante qu'elle représente. De plus, avec le rejet de l'ennemi «intérieur», du «frère ennemi», on se situe de plus en plus dans une logique de guerre civile: au fur et à mesure que la distance géographique qui sépare de l'ennemi se réduit, les critères de distinction deviennent plus flous, et l'acharnement à le détruire plus féroce.

Que ce soit dans l'évolution des fronts ou dans la définition de l'ennemi, on voit à l'œuvre une décomposition progressive des anciennes communautés d'appartenance - la Monarchie, la loyauté commune envers le Roi - et une recomposition simultanée de leurs critères de distinction. Or, plus on avance dans ce processus de décomposition, plus les distinctions communautaires deviennent floues. La première césure, fondamentale, est de nature idéologique: elle distingue les partisans du statu quo, celui de l'attachement à la péninsule - voire à l'absolutisme - et ceux de la réforme, allant dans le sens de l'autonomie des pueblos et d'une révolution libérale. Cette distinction acquiert rapidement un contenu géographique, opposant, d'une part, les deux rives de la Monarchie (Américains contre péninsulaires), et dressant, d'autre part, le Río de la Plata contre les régions voisines (Buenos Aires contre Montevideo et contre Lima). Une seconde césure oppose les dirigeants de Buenos Aires au chef de la Bande Orientale. Il s'agit cette fois d'une lutte entre patriotes, au cours de laquelle les critères de distinction passent par des choix concernant l'avenir de la nouvelle communauté. La guerre n'a donc pas pour seul effet de dessiner les contours de la nouvelle appartenance politique; elle en définit également le contenu.

21 Bien que le terme de «fédéralisme» soit couramment employé à l'époque, il désigne ce que l'on entend à l'heure actuelle par «confédération», une alliance souple de provinces souveraines et autonomes. Cf. González, 1955. Reyes Abadie, 1975. Petit Muñoz, 1988. 


\section{LA GUERRE COMME MOTEUR DE L'ORGANISATION POLITIQUE}

Du fait de ses multiples aspects 22 , la crise de la Monarchie a pour effet de brouiller, dans l'ensemble du monde hispanique, les pistes de la légitimité. Dans les royaumes américains, le droit des cités à assumer la souveraineté au nom du Roi captif entre en concurrence avec le maintien en place des fonctionnaires royaux, qui exercent leur autorité au nom du monarque. La formation de la Junte de Buenos Aires, qui se substitue à l'autorité du vice-roi Cisneros le 25 mai 1810, représente le choix de la première solution. Mais elle ne tarde pas à s'affronter au vice-roi Abascal qui lui, représente la deuxième. Le conflit de légitimité qui oppose les deux instances ne peut donc se résoudre que par les armes. La guerre est donc, dès le début du processus révolutionnaire, un instrument de légitimation du nouveau pouvoir.

Dans le cas du Río de la Plata, ce processus de légitimation trouve son origine dans les invasions anglaises de 1806-1807, qui révèlent la capacité d'une ville de 1'Empire à assurer sa propre défense face à une attaque extérieure ${ }^{23}$. Du fait de la relative faiblesse de l'appareil militaire - malgré les efforts entrepris par la Couronne dans le cadre des réformes bourboniennes - les vecinos de Buenos Aires, à l'initiative du cabildo, lèvent des bataillons de milices qui leur permettent de remporter la victoire à deux reprises, assurant ainsi la sécurité de toute la vice-royauté. Les conséquences politiques de cet épisode sont multiples. Tout d'abord, la cité acquiert son autonomie sur le plan militaire et politique. La mobilisation s'accompagne en effet de la destitution du vice-roi Sobremonte et de l'élection de son successeur, le capitaine Jacques de Liniers, qui prend le commandement des troupes. De plus, cette mobilisation favorise l'apparition de pratiques «démocratiques» au sein de la plèbe urbaine, qui vont être à l'origine de la diffusion du modèle dans des secteurs plus larges de la société24. Enfin, les villes de l'Intérieur sont appelées à participer en envoyant des contributions en hommes et en argent. Il se crée de ce fait un lien de solidarité entre ces cités et Buenos Aires, qui renforce la conscience d'une appartenance commune et l'obédience liant les cités à la capitale, celle-ci s'attribuant à l'issue de la victoire le rôle de rempart de la vice-royauté. Par ses exploits militaires, Buenos Aires a prouvé sa capacité à se passer de la métropole si celle-ci faisait défaut, et d'assurer seule la gestion de son gouvernement.

Ce précédent est largement exploité au moment de la révolution, tant sur le plan symbolique que sur le plan stratégique. Pour se faire accepter par les

22 Portillo Valdés, 2000: 159-256.

23 Gallo, 2004.

24 González Bernaldo, 10 (Paris, 1990): 177-195. Halperín Donghi, 1978: 121-158; 1982. 
autres cités, la Junte utilise à son profit la légitimité acquise par la capitale en 1806-1807, jouant sur l'urgence d'une décision à prendre dans un contexte où le Río de la Plata est en péril, menacé par des armées étrangères, celles de Napoléon ou celles du Portugal. Buenos Aires se présente à nouveau comme le rempart et le guide de la vice-royauté, invitant les autres cités à suivre son exemple. Retrouvant les accents de 1807, le cabildo évoque ainsi «l'honneur d'une cité qui a été et demeure le centre de la loyauté et de l'héroïsme, et a consenti à de nombreux sacrifices pour conserver l'intégrité des droits de notre auguste monarque.... ${ }^{25}$. L'autre facette de ce processus est le rôle des milices urbaines dans la formation de la Junte du 25 mai et de l'Expédition Auxiliaire. Mieux connu, ce point n'en est pas moins capital: de juin à août 1810 , c'est une véritable course de vitesse à laquelle se livre le nouveau pouvoir face à Abascal. S'il s'agit en grande partie d'une guerre des mots, il est indéniable que la présence d'hommes en armes joue à plein dans la décision des cités de reconnaître la Junte de Buenos Aires. À cet égard, la comparaison avec d'autres régions comme le Chili ou la Nouvelle-Grenade se révèle, une fois de plus, éclairante: à la différence de Santiago ou de Santa Fé de Bogotá, incapables de s'imposer face aux prétentions souveraines des autres cités, Buenos Aires a les moyens symboliques et surtout militaires d'emporter l'adhésion des autres et d'exercer la représentation de l'ensemble. L'Expédition Auxiliaire apparaît donc comme le bras armé de la Junte, chargé de réaliser son programme: destituer les anciennes autorités, imposer le nouveau pouvoir et faire élire des représentants pour le congrès prévu par la Junte.

Au-delà de ce recours à la force, ce sont des arguments juridiques — essentiellement la thèse de la rétrocession de la souveraineté aux pueblos — qui fondent la légitimité de la Junte de Buenos Aires à assurer «la conservation des droits du souverain» et à diriger l'ensemble de la vice-royauté. Mais au fur et à mesure que s'accentue la rupture avec la péninsule, c'est la guerre qui renforce la légitimité des détenteurs du pouvoir suprême, lequel passe de plus en plus des mains des «docteurs» aux mains des militaires. Cette tendance s'amorce avec Carlos de Alvear, jeune et brillant officier qui rentre d'Espagne en 1812, en même temps que son compagnon et rival San Martín. Fils de famille, disposant dans la capitale d'appuis solides et variés, Alvear joue un rôle très actif au sein de l'Assemblée Générale Constituante (1813), dont il est le secrétaire. Il est à l'origine de la création du Directoire en 1814, concentration

25 Oficio del Cabildo de Buenos Aires al de Tucumán, 29 de mayo de 1810, in Freyre, 1909: 70 .

Revista de Indias, 2009, vol. LXIX, n. ${ }^{\circ} 246,17-44$, ISSN: 0034-8341 doi: 10.3989/revindias.2009.011 
du pouvoir suprême aux mains d'une seule personne, dont son oncle, Antonio Posadas, est le premier détenteur.

La même année, Alvear prend la tête de l'armée de la Bande Orientale et met un terme victorieux au blocus de Montevideo. C'est ici qu'apparaît la fonction de légitimation qu'assurent les victoires militaires. La célébration de cette victoire ${ }^{26}$ est ordonnée par le pouvoir, dans une circulaire accompagnée du récit de la bataille, dû à la plume d'Alvear. À cette occasion, les félicitations envoyées au Directeur Suprême allient les vertus du militaire à celles du chef d'État, comme si l'oncle et le neveu se confondaient en une seule et même personne. Le cabildo de Tucumán déclare ainsi à Posadas que «sa respectable personne unit la valeur et la compétence militaire de Washington à l'habileté, la prévoyance et la prudente sûreté de Franklin» ${ }^{27}$. En réalité, le prestige rejaillit tout entier sur Alvear, préparant son accession au pouvoir quelques mois plus tard. Sous son règne, le régime directorial atteint l'apogée de sa dérive autoritaire et centraliste, ce qui entraîne le coup de force de Fontezuelas, perpétré par les officiers de l'armée de Buenos Aires, et sa destitution en 1815.

L'autre exemple est celui du général Rondeau. Militaire de carrière, celui-ci commande les troupes de la Bande Orientale dès 1811 et dirige le siège de Montevideo en 1812. Laissant ensuite la place à Alvear, il prend le commandement de l'Armée du Nord. Élu Directeur Suprême après la chute d'Alvear en 1815, il choisit de rester sur le front, le colonel Alvarez Thomas exerçant son mandat en qualité de suppléant. Après la défaite de Sipe Sipe en novembre, il est destitué par le nouveau Directeur, Pueyrredon, qu'il remplacera une nouvelle fois en juin 1819.

Alvear offre l'image d'une tentation césariste, celle du jeune officier à l'ascension rapide, soucieux de son image de marque et entièrement préoccupé par la conquête du pouvoir. Le cas de Rondeau est d'une autre nature: s'il révèle comme le précédent l'immixtion profonde des sphères militaire et politique - de fait, toute distinction semble impossible tant la construction de l'État apparaît inséparable de sa défense - il incarne le recours suprême dans une situation de vide de pouvoir. Le fait que Rondeau commande les principales armées du pays et accède par deux fois à la magistrature suprême

26 En réalité, ce sont plusieurs victoires qui sont fêtées les unes à la suite des autres: la victoire navale de l'Amiral Brown au Buceo, le 14 mai 1814, la prise de Montevideo proprement dite, du 20 au 23 juin, puis les victoires d'Alvear sur Otorgués, le lieutenant d'Artigas, au Río de la Piedras le 24 juin et à Marmarajá le 8 octobre.

27 Oficios del Gobernador y del Cabildo de Córdoba al Director Supremo, 2 de julio de 1814, Archivo General de la Nación (AGN), Gobierno de Córdoba, Sala X, legajo 5-2-7. 
révèle, outre la relative pénurie d'hommes capables d'exercer ces charges, la confiance que lui vouent les responsables politiques. Son rôle à la tête des armées en fait le défenseur de la révolution, le protecteur naturel des pueblos, et, par là même celui qui possède la plus grande légitimité pour les gouverner. Cela apparaît clairement en 1815, lorsque les gouverneurs des provinces, ignorant que Rondeau a déjà été nommé Directeur Suprême, se tournent vers lui en tant que chef de l'Armée du Nord, «dépositaire de la force qui doit garantir notre justice», selon l'expression du gouverneur de Córdoba ${ }^{28}$. C'est donc la reconnaissance d'une autorité supérieure chez les chefs militaires, en lien avec la défense de la révolution, qui leur permet d'intervenir dans la sphère du pouvoir.

Ce qui vient d'être dit des hommes s'applique également à leur politique: la poursuite de la guerre, confondue avec la défense de la révolution, constitue dès 1812 la justification suprême des orientations prises pour la construction institutionnelle. Si les rapports entre armée et pouvoir central évoluent, cela provient de ce que la place de la guerre dans la définition même de la révolution s'est inversée par rapport à 1810. Au début de la révolution, le projet de rénovation du pouvoir et de la société devait s'imposer par l'adhésion des cités, obtenue, le cas échéant, par la guerre. L'armée n'était qu'un des moyens de suppléer au discours pour imposer ce projet révolutionnaire. En 1815-1816, du fait de la carence du processus d'institutionnalisation, la construction d'un régime politique se trouve, à l'inverse, entièrement subordonnée à l'impératif militaire.

Durant ces années, la guerre justifie en particulier la pratique du provisoriato, c'est-à-dire l'ajournement d'un véritable régime représentatif, qui aurait permis de doter le pays d'une Constitution stable ${ }^{29}$. Cette tendance est inaugurée en 1813, avec l'ouverture de l'Assemblée Générale Constituante. Inaugurée dans un contexte militaire favorable, après les brillantes victoires de Belgrano, l'Assemblée connaît pendant plusieurs mois une importante activité législative. Sa souveraineté est alors pleinement respectée, et l'existence de plusieurs projets constitutionnels autorise à penser que l'institutionnalisation de la révolution est en cours. Tout change avec les défaites de Vilcapugio et Ayohuma, en octobre: ces revers provoquent et justifient une crispation du pouvoir exécutif, qui concentre à nouveau le pouvoir entre ses mains. Les sessions de l'Assemblée sont bientôt suspendues, et son rôle réduit à celui d'une chambre d'enregistrement des décisions du pouvoir suprême.

28 Oficio de José Javier Díaz a Rondeau, 23 de abril de 1815, in Segreti, 1966, vol. 2: 476.

29 Verdo, 31 (Bilbao, 2006): 513-536. 
C'est à cette période que le provisoire devient la principale caractéristique du gouvernement. L'argument sans cesse invoqué pour expliquer le report du processus d'institutionnalisation est celui de la guerre, comme si seule la victoire conférait au régime une légitimité nécessaire pour se doter d'institutions indépendantes. Ce problème, bien réel, en cache un autre, celui de la définition du nouvel État. Les dirigeants se refusent à adopter une Constitution tant que la représentation des Provinces Unies ne sera pas complète. Or, la vice-royauté du Río de la Plata comprenait les provinces de la Bande Orientale et du Haut Pérou, désormais isolées par la guerre. Posé une première fois en 1813, le problème se répète dans les mêmes termes après la déclaration d'indépendance de 1816, lorsqu'il s'agit de statuer sur la forme du régime. Alors que des députés du Haut Pérou siègent au congrès de Tucumán, l'incomplétude de la représentation et la poursuite de la guerre sont invoquées par les députés de Buenos Aires pour lutter contre la tendance majoritaire, celle favorable à l'érection d'une monarchie constitutionnelle respectant l'autonomie des différentes provinces.

Toutefois, le ralliement des députés aux options «centraliste» ou «autonomiste» répond tout autant à un problème de stratégie militaire qu'à une logique d'affrontement entre Buenos Aires et l'Intérieur. La construction de l'État est en effet subordonnée aux visées géostratégiques des dirigeants, qui ont pour objectif les mines de Potosí et le port de Montevideo. Elle est également liée au contexte général de l'Amérique: à partir de 1814, la conjoncture change avec le retour de Ferdinand VII, le rétablissement de l'absolutisme en Espagne, la reconquête royaliste au Chili et les attaques d'Artigas dans le nord-est. La révolution se sent menacée et le régime tend à devenir de plus en plus autoritaire. Ce contexte difficile, surtout après la défaite de Sipe Sipe, explique le choix délibéré du centralisme à la fin de 1816. La désignation de Pueyrredon comme Directeur résulte de la pression exercée sur les députés par les militaires, notamment San Martín. En effet, seul Pueyrredon paraît à même de rallier l'adhésion des provinces tout en préservant le caractère centraliste du régime, nécessaire à la réussite $\mathrm{du}$ «plan continental» $»^{30}$. Le principal objectif du régime consiste en effet à soutenir cette opération militaire de grande envergure. De fait, le nouveau Directeur se transforme en «commis voyageur de la guerre», passant les deux premiers mois de son mandat à aller visiter sur le terrain les principaux chefs militaires (notamment Güemes et San Martín),

30 Elaboré par San Martín dans les années 1814-1815, celui-ci consiste à attaquer les royalistes en passant par le Chili, puis à rejoindre Lima par la mer. Le Haut Pérou cesserait alors d'être le front principal pour devenir une simple marche, destinée à contenir les troupes royalistes (Ramos Pérez, 1988). 
afin de s'entendre avec eux sur un plan d'ensemble, coordonné et appuyé par la capitale.

La guerre permet par là même de maintenir pendant dix ans l'adhésion des cités à la domination de Buenos Aires. Celle-ci est en effet la directrice des opérations militaires, et la principale pourvoyeuse d'argent, de vivres, d'hommes et de montures. Quant à l'union des provinces, elle est indispensable à la poursuite du combat. La guerre d'indépendance est donc le fondement du lien politique et la cause commune, en lieu et place d'une organisation constitutionnelle.

\section{LA GUERRE ET L'ÉMERGENCE DES ÉTATS PROVINCIAUX}

Pourtant, le conflit va également être à l'origine de la maturation de la forme étatique dans les provinces. S'installant comme une réalité permanente dans les cités de l'intérieur, il provoque une mobilisation intense des cités qui se révèle être le principal facteur de gestation des États provinciaux ${ }^{31}$.

La mobilisation de l'Intérieur, par le biais des recrutements et de contributions, commence dès la mise en route de l'Expédition Auxiliaire. À partir de la stabilisation des fronts en 1812, les cités sont placées au centre d'un vaste dispositif d'approvisionnement des troupes, destiné à soutenir le double effort mené contre les royalistes, dans le Haut Pérou et la Bande Orientale. L'organisation d'ensemble est coordonnée par le pouvoir central, notamment en ce qui concerne le recrutement. Les troupes levées dans l'Intérieur sont plutôt destinées à l'Armée du Nord, celles de la Bande Orientale provenant des provinces de Buenos Aires et du Littoral. Aux anciens corps de milices s'ajoutent les nouvelles compagnies civiques créées par les cabildos, ainsi que les troupes levées par certains vecinos. Si les vagabonds, les gens sans emploi et les esclaves en fuite sont recrutés en priorité pour ne pas priver la province de ses bras, des circonstances exceptionnelles imposent parfois la levée en masse, comme à Salta en août 1812. Il se produit alors, à travers la mobilisation, un brassage social d'ampleur inégalée qui contribue à renforcer l'identité patriotique à tous les niveaux où celle-ci se décline, principalement provinciale et américaine ${ }^{32}$.

Pour assurer l'équipement et la subsistance des soldats, le soutien de la communauté se révèle indispensable. Les contributions en argent et en nature sont le complément naturel du recrutement des troupes. Si les donations spon-

31 Sur le concept et la genèse des États provinciaux, cf. Chiaramonte, 1995: 167-205.

32 Chiaramonte, 1 (Buenos Aires, 1989): 71-91. 
tanées affluent en 1810, comme sous les invasions anglaises, celles-ci cessent rapidement et sont remplacées par les réquisitions et les emprunts forcés, des «plans de contribution» à destination des cités étant régulièrement mis en place par le pouvoir central. On peut citer comme exemple le vaste plan d'approvisionnement mis en place dans le Nord-ouest en 1813-1814, les provinces appelées à fournir des vivres, des toiles, des chaussures, des armes, des munitions et des montures. Le pouvoir central, qui n'est pas en mesure d'avancer les fonds, confisque en effet les ressources des cités pour financer la guerre: en juillet 1813, les cabildos reçoivent l'ordre de transférer les fonds du ramo de sisa dans les caisses de l'État; en décembre 1814, ils doivent livrer un état complet de leurs ressources et de leurs rentes avec les provenances et méthodes de gestion. Face à ces ponctions régulières, les cabildos en vain plaident la cause de leur cité.

Qu'on y consacre ses biens ou sa personne, la mobilisation concerne donc toutes les couches sociales. La guerre ouvre la sphère de participation des populations à la «cause commune» beaucoup plus sûrement que ne le font les élections. Elle contribue par ailleurs à multiplier les liens entre la campagne de la cité et à intégrer les deux espaces dans un même univers, transformant la notion même de «province» ${ }^{33}$. Cette mobilisation d'ampleur inégalée a des répercussions essentielles sur le plan politique, qui se font de plus en plus sentir à partir de 1815. À la suite de la défaite de Sipe Sipe, l'effort de guerre est encore accru dans les cités de l'Intérieur: en lien avec le «plan continental» de San Martín, Guëmes parvient à réunir une armée de 6000 hommes et impose une contribution forcée pour payer les uniformes et le ravitaillement. Tucumán est dans un état de banqueroute permanent qui l'oblige à taxer les vecinos. Outre la nécessité de ravitailler l'armée, la cité est dans l'obligation d'accueillir les blessés, ainsi que le reste de la troupe après la défaite de Sipe Sipe: au total, quelques 1500 personnes que les autorités de la cité ont en charge de loger, nourrir, vêtir et équiper. L'effort financier repose essentiellement sur les commerçants, qui sont de plus en plus réticents à payer. Quant à Mendoza, elle donne l'exemple d'une province entièrement mobilisée pour mettre sur pied l'Armée des Andes. L'essentiel de l'équipement est fabriqué sur place et financé par des expédients divers: impôts et taxes nouvelles, contributions volontaires, emprunts forcés, réduction de la solde des agents de l'État, confiscations de biens et réquisitions. Comme le déclare avec fierté le cabildo de Mendoza en avril 1816:

33 À la fin de l'ancien régime, la province comprend la cité et sa juridiction (comarca), dont elle assure le gouvernement et la représentation. À l'issue de la révolution, l'ensemble forme un espace beaucoup plus intégré sur le plan matériel et politique. La représentation politique de la campagne est alors acquise. 
«Il est impossible de donner une idée exacte de toutes les dispositions, des efforts consentis en préparatifs de guerre et des moyens économiques projetés pour mener à bien une si grande œuvre, sans autres ressources que les maigres entrées du Trésor (quasiment réduites à néant par la cessation du commerce avec le Chili) et les contributions par lesquelles les vecinos ont participé aux efforts héroïques de leur gouverneur» ${ }^{34}$.

Cette mobilisation de grande ampleur résulte désormais d'initiatives prises à l'échelle des provinces, combinant les ressources des cités et des campagnes, donnant par là même plus de vigueur à l'affirmation d'une identité provinciale. De fait, à travers la correspondance des gouverneurs, on s'aperçoit que Buenos Aires a de plus en plus de mal à les soutenir financièrement: elle ne donne, par exemple, que 6000 pesos à Mendoza pour équiper l'Armée des Andes alors que la contribution des vecinos représente quatre fois plus. Le pouvoir central perd donc en prestige dans la mesure où il peut de moins en moins soutenir l'effort de guerre.

En second lieu, à partir de 1815, l'armée s'impose comme un acteur à part entière de la souveraineté. Dotés d'une capacité d'initiative politique (dont ils font preuve lors du soulèvement de Fontezuelas), les officiers apparaissent comme une alternative crédible face aux dirigeants impopulaires et se font les champions des droits des cités. On a vu qu'au moment de la chute d'Alvear, Córdoba et Salta se plaçaient ainsi spontanément sous la protection du général Rondeau. Durant les années suivantes, l'armée est non seulement un élément à part entière du jeu politique, comme le montre la signature de pactes entre elle et les provinces, mais elle joue de plus un rôle essentiel au sein des communautés. Dans les régions du mobilisées pour l'effort de guerre, la figure du citoyen-soldat ${ }^{35}$ s'impose comme fondement de l'identité provinciale, tandis que les militaires trouvent leur place dans la vie politique soit comme citoyens, soit comme représentants de la cité. Cette évolution est liée à l'importance grandissante des gouverneurs, dont les responsabilités militaires augmentent. Comme nous l'avons vu, ils ont à charge de s'occuper du financement de la guerre, du logement, du ravitaillement, de l'entretien de la troupe, ainsi que du paiement des soldes. Du reste, la plupart sont également officiers, tant et si bien qu'à partir de 1815 , l'administration des provinces est pratiquement confondue avec celle des armées. Ces personnages sont de plus en plus autonomes par rapport au pouvoir central, surtout à partir du moment où ils créent des armées dans le cadre des provinces, comme le font Güemes à Salta

\footnotetext{
34 Oficio del cabildo de Mendoza al Congreso, 24 de abril de 1816, AGN, Comunicaciones Congreso-Gobierno 1816-1819, Sala X, legajo 3-9-4.

35 Hébrard, 1996.
} 
ou San Martín à Mendoza. Leur autonomie et leur ascendant sur les populations locales sont puissamment confortés par le caractère populaire de leur élection, établi par le Statut provisoire de 1815. À cette date, les gouverneurs ne sont plus les représentants du pouvoir de la capitale, parachutés dans les cités, mais des représentants naturels de leurs communautés.

Enfin, la guerre a pour effet de multiplier les relations entre les provinces, qui ne transitent plus nécessairement par Buenos Aires. Les gouverneurs suivent de près la situation des fronts, organisent les convois et se tiennent au courant des péripéties politiques dans les régions voisines. Ces relations donnent naissance à une nouvelle solidarité entre les cités, une géographie patriotique organisée autour des lieux d'où partent les expéditions militaires. Le fossé qui apparaît entre Buenos Aires, accaparée par les tensions dans le Littoral, et les cités andines ne fait que se creuser à la faveur du «Paso de los Andes» en 1817. Celui-ci constitue un moment essentiel de la geste révolutionnaire: le projet militaire de San Martín prend alors le pas sur celui du Directoire. Comme le montrent les cérémonies patriotiques, c'est dans la campagne des Andes que la Révolution de Mai trouve un second souffle. Cette construction s'ancre dans un imaginaire patriotique où la guerre a peu à peu pris le pas sur la révolution. Depuis 1812, les victoires militaires constituent le principal fondement d'une identité collective, servant à la fois à raffermir le prestige des cités victorieuses et à créer une cause commune où toutes se sentent partie prenante ${ }^{36}$. L'organisation concertée à laquelle elles sont contraintes développe une conscience de posséder et de forger une destinée commune, où les nécessités et les valeurs de la guerre supplantent les réformes et les principes libéraux comme creuset de l'identité patriotique.

\section{LA GUERRE ET LA RECOMPOSITION DE LA SOUVERAINETÉ}

L'aboutissement de ce processus est la proclamation de l'indépendance par les différentes provinces, au cours des années 1820 et 1821 . Consolidées sur le plan de leur organisation territoriale et matérielle, les cités se soustraient à la domination de Buenos Aires désormais incapable de mener la guerre sur plusieurs fronts. Toutefois, malgré leur ferme intention de défendre la souveraineté dans un cadre provincial, les cités conservent entre elles un lien destiné à refonder l'union politique et à poursuivre l'effort de guerre. C'est en effet la guerre qui rend nécessaire le maintien et l'institutionnalisation d'un cadre commun, aucun gouverneur n'étant en mesure de lever à lui tout seul une ar-

36 Verdo, 2006: 174-179. 
mée suffisante pour affronter les royalistes. Se pose alors le problème du rapport entre projet militaire et organisation politique: la création institutionnelle doit-elle délibérément prendre le pas sur la guerre ou doit-on se plier aux urgences du moment, quitte à prolonger le provisoriato?

Loin de remettre en cause leur dévouement envers la «cause commune» et les armées «nationales», la proclamation de l'indépendance et le choix, par les cités, du système «fédéral» (c'est-à-dire de la souveraineté provinciale) représente un nouvel élan de la coopération avec les chefs militaires. Le plan que le gouverneur de Córdoba, Bustos, expose à Güemes le 3 février $1820^{37}$ vise à organiser la participation de toutes les provinces pour augmenter les effectifs de l'Armée du Nord, qui rejoindrait celle des Andes pour marcher définitivement sur Lima. Pour lui, la réussite de ce projet militaire dépend d'une solution politique: en promouvant le système fédéral, il s'agit de canaliser l'aspiration autonomiste des cités afin de construire un gouvernement central doté de suffisamment d'autorité pour conduire la guerre.

Bustos n'ignore pas quelles sont les vues de son interlocuteur: pour Güemes, la guerre est une priorité absolue dans la réorganisation politique en cours. Le congrès que Bustos envisage de réunir est pour lui un moyen d'obtenir la participation des provinces à l'expédition qu'il entend mener vers le Pérou: selon lui, les députés devront inviter les provinces à se «fédérer en guerre (sic) contre les ennemis de l'indépendance et de la liberté civile» ${ }^{38}$. L'organisation de la force militaire, l'entretien des troupes et toutes les décisions relatives au combat seraient l'affaire commune des provinces qui nommeraient, entre autres, les officiers. La gestion de cette armée ne devrait en aucun cas être mêlée aux affaires civiles: c'est sur ce point que Güemes se démarque de Bustos, pour qui l'administration des provinces est étroitement liée à la conduite de la guerre.

Peu après, en avril 1820, le cabildo de Salta lance un appel aux autres cités pour qu'elles l'aident à repousser une invasion royaliste (la septième en dix ans!), dirigée par le général Ramírez de Orozco. Tandis que la cité de Salta fournit, une fois de plus, un effort considérable, les autres provinces lui accordent un soutien important en hommes et en argent, vivres et montures. Seule Buenos Aires, en proie à une vive instabilité politique, refuse son aide au motif qu'elle doit lutter contre Santa Fé. Ce refus signifie clairement qu'elle privilégie désormais ses intérêts plutôt que la «cause commune», renonce à être

\footnotetext{
37 Oficio de Bustos a Güemes, 3 de febrero de 1820, in Güemes, 1977-1990, vol. 6: 41.

38 Instrucción para los dos diputados o representantes que van de esta ciudad de Salta al congreso que se invita a la de Córdoba, 18 de marzo de 1820, AGN, Gobierno de Salta, Sala VII, legajo 10-3-3.
} 
impliquée dans une guerre lointaine et préfère se défendre contre ses plus proches ennemis.

Malgré cette défection, l'invasion royaliste est victorieusement repoussée à la mi-juin. L'épisode montre bien que malgré le choix de l'autonomie, la lutte contre les royalistes demeure le principal élément d'une identité commune entre les provinces. Un mois plus tard, San Martín confie à Güemes le soin d'organiser une expédition de renfort pour repousser les royalistes vers Lima. Cette nouvelle responsabilité amène Güemes à envisager de réunir dans l'urgence un congrès pour recruter des soldats et collecter les ressources nécessaires à la tenue de l'expédition. Une fois encore, Buenos Aires reste très réticente, tandis que Bustos, bien qu'en désaccord avec Güemes au sujet du congrès, appuie entièrement le projet d'expédition. Il rappelle toutefois que l'Armée du Nord, «qu'[il] a entretenue [en tant que général et gouverneur] avec les ressources de Córdoba, n'appartient pas à cette province mais à l'État en général» ${ }^{39}$. En revanche, le manque de coopération d'Aráoz, gouverneur de Tucumán, fait échouer le projet de Salta au début de l'année 1821 et précipite le conflit entre les deux provinces. La même année, Güemes, désavoué par les autorités de Salta, trouve la mort au cours d'une embuscade.

Refusant de suivre la province de Salta qui, après la mort de Güemes, a signé un armistice avec les royalistes, les chefs patriotes consentent un dernier effort, encouragés par l'annonce du débarquement de San Martín à Lima au mois de septembre 1821. Le nouveau gouverneur de Tucumán, Abraham González, reprend à son compte le projet d'expédition vers le Haut Pérou et adresse une circulaire aux autres provinces, en accord avec les plans de San Martín et O'Higgins. Comme Bustos, il considère ce projet comme le fondement de sa légitimité politique en tant que gouverneur. Toutefois, il considère que la guerre se situe en amont de la question politique, celle-ci ne devant être abordée qu'une fois la paix rétablie. Pour González, comme c'était le cas pour Güemes, la victoire est donc le préalable indispensable à l'institutionnalisation du régime ${ }^{40}$. Mais il ne parvient pas plus que Güemes à mobiliser l'ensemble des provinces, victimes de leurs dissensions internes et de l'absence d'une autorité supérieure. De fait, malgré ce qu'en pensaient les chefs militaires les plus volontaristes, la guerre se révèle impossible à mener sans organisation politique préalable.

Le problème de la guerre se pose aussi d'une autre façon à cette période, puisqu'elle oppose entre elles, dès leur formation, les provinces souveraines 48.

39 Oficio de Bustos a Güemes, 21 de diciembre de 1820, in Güemes, 1977-1990, vol. 6:

40 Fitte, 20 (Buenos Aires, 1976): 177-203. 
du Río de la Plata: provinces du Littoral contre Buenos Aires, Salta contre Tucumán. On voit alors d'autres cités (notamment Córdoba) jouer le rôle de conciliateur et d'arbitre, tandis que les traités ou pactes inter-provinciaux ${ }^{41}$ font leur apparition, avant de s'imposer comme l'un des instruments privilégiés de la construction nationale.

Le premier de ces textes est le traité du Pilar, signé à l'issue de la bataille de Cepeda le 23 février 1820 entre les nouvelles autorités de Buenos Aires et les gouverneurs du Littoral. Entérinant les vues des vainqueurs, le premier article prend acte du «vœu que la nation a prononcé et proclame l'avènement de la "fédération" $\rangle^{42}$. Le texte prévoit en outre convocation d'un congrès à San Lorenzo, dans la province de Santa Fé, pour jeter les bases d'un nouveau gouvernement. Il fixe enfin les modalités de la paix: cessation des combats, retrait des troupes, libération des prisonniers, épuration de l'administration centraliste. Buenos Aires conserve la liberté du commerce des armes et des munitions, ainsi que la liberté de la navigation sur les fleuves Paraná et Uruguay, atout majeur de son commerce. On voit donc que ce texte, au-delà du traité de paix, a une profonde portée politique: il sanctionne la fin de la domination de Buenos Aires sur l'ancienne vice-royauté et modifie en profondeur l'organisation politique du Río de la Plata, par le choix du système fédéral et la convocation du congrès. Enfin, il révèle que les provinces, signataires du pacte, sont désormais les nouveaux acteurs de la souveraineté.

Après la reprise des combats et la victoire de Pavón en août de la même année, Bustos envoie des émissaires auprès de Buenos Aires et du Littoral au début du mois d'octobre ${ }^{43}$. Le but de la mission consiste à ramener la paix pour permettre l'organisation politique des provinces; il s'agit d'obtenir un cessez-le-feu, de rétablir la libre circulation des marchandises et de faire nommer des députés au Congrès. Accueillie avec soulagement par les belligérants épuisés, l'initiative se conclut le 24 novembre par la signature du pacte de Benegas, qui reprend les orientations du Pilar. Les relations entre Buenos Aires et Santa Fé seront désormais fondées sur «la paix, l'harmonie et la bonne correspondance» ${ }^{44}$; un congrès se réunira dans les deux mois, devant lequel seront portés les différends entre les provinces; les autres dispositions d'ordre militaire étant placées sous la garantie de Córdoba. Par sa médiation, celle-ci contribue donc à refonder l'ordre politique et reçoit les félicitations des autres entités souveraines.

\footnotetext{
41 Gutiérrez Ardila, 2008.

42 Demicheli, 1955, vol. 1: 449-452.

43 Segreti, 18 (La Plata, 1968): 225-242.

44 Demicheli, 1955, vol. 1: 453.
} 
Au même moment, la situation se tend sur le front du Nord-ouest. À la suite de l'échec de l'expédition de renfort à San Martín, les relations se dégradent entre Güemes et le gouverneur de Tucumán, Aráoz, qui lui a refusé son aide. L'intervention d'Aráoz dans la province voisine de Santiago del Estero, en janvier 1821, sert de prétexte à Güemes pour attaquer celle de Tucumán. Mais, fait remarquable, les pourparlers de paix commencent presque en même temps que les hostilités: avec des accents pathétiques faisant allusion à sa propre expérience, Buenos Aires appelle Tucumán et Santiago à se réconcilier et nomme un émissaire, tentant par là même de retrouver sa place dans le jeu politique. Dans le même temps, Aráoz entame les pourparlers de paix avec Santiago puis avec Salta. Córdoba envoie également un médiateur, récidive de la mission du Littoral, et y ajoute un message de la part des députés réunis à Córdoba: «Assez de sang et de désolation, unissons-nous de bonne foi et forgeons derechef un pays si digne de le devenir» ${ }^{45}$. Les négociations entre Aráoz et Santiago del Estero amènent à la signature du pacte de Vinará le 5 juin 1821, sous les auspices de Córdoba: rétablissement de la paix, établissement d'une défense mutuelle entre les «provinces-sœurs», rétablissement des communications et du commerce, union fraternelle entre Tucumán, Santiago et Salta contre le nouvel ennemi commun, Güemes, qui vient d'être déposé par le cabildo de Salta.

La déposition de Güemes permet de le désigner comme seul responsable des événements et de justifier, par le cabildo, la signature de l'armistice avec les royalistes. Outre le fait que l'ancien gouverneur soit accusé de tous les maux, son administration est décrite dans les mêmes termes que la guerre civile, comme la négation même de l'ordre politique, la plus grande menace contre la cohésion de l'ordre social. À l'inverse, pour la première fois depuis 1810, l'ennemi royaliste apparait «davantage comme un père et un protecteur que comme un tyran» ${ }^{46}$. Par là même, les vecinos de Salta s'efforcent de se persuader qu'en concluant l'armistice, ils sont parvenus à sauver leur honneur patriotique, mis à mal par un gouverneur devenu despote. On voit également affleurer ici le lien subtil qui existe pour les acteurs entre la construction politique et la guerre: loin d'être de nature différente, les deux états constituent en fin de compte les deux extrêmes d'une même situation. Dans un cas la confection d'un ordre qui garantit la solidité de l'édifice (ou la vitalité de l'organisme), dans l'autre la lutte mortelle de toutes les parties entre elles, menant à l'anomie.

45 Oficio enviado por los diputados al Congreso a los gobiernos beligerantes, 28 de marzo de 1821, AGN, Gobierno de Córdoba, Sala X, legajo 5-4-1.

46 Oficio del cabildo de Salta al de Buenos Aires, 21 de julio de 1821, in Güemes, 1977-1990, vol. 11: 311-314. 
En septembre, Aráoz est déposé par Abraham Rodríguez pour le même motif, celui d'avoir trahi, à son profit personnel, ses compatriotes et la cause de la Patrie. Les nouvelles autorités célèbrent la «glorieuse révolution du pueblo opprimé» par la tyrannie, incarnée par la guerre contre les provinces sœurs de Santiago, Catamarca et Salta. Là aussi, la guerre civile est condamnée en ce qu'elle a d'incompatible avec les principes défendus depuis 1810. L'autoritarisme des chefs militaires est une nouvelle fois dénoncé; on les accuse d'avoir tenté de «s'opposer comme des mandones à des frères ou à des parents» ${ }^{47}$. Il est significatif de voir ici réapparaître le terme employé au début de la révolution pour désigner les agents de la Couronne, opposé au registre de la fraternité, du lien naturel entre les provinces souveraines. La chute des caudillos, nouveaux despotes, représente une nouvelle avancée dans la révolution des pueblos. Le cabildo de Tucumán déclare ainsi que le système politique qu'ils ont tenté de promouvoir n'était qu'un «incroyable féodalisme, recouvert d'un voile de fédéralisme». Y est opposée la nouvelle marche des pueblos vers l'indépendance et la liberté, obtenue par la pratique «de l'amitié et de l'union», c'est-à-dire la paix civile ${ }^{48}$.

Au terme de cette étude, il apparaît donc que la guerre possède, au Río de la Plata, une dimension politique essentielle, qui se décline de plusieurs façons. Permettant de désigner un ennemi, elle contribue, tout d'abord, à définir la communauté que les dirigeants révolutionnaires s'efforcent de faire advenir: communauté politique, par son choix de l'autonomie et du libéralisme, puis communauté «nationale», par son rejet de l'Espagne. Au de cet ensemble, la vindicte jetée sur tel ou tel dessine de plus en plus précisément les contours du projet politique des dirigeants porteños. En second lieu, la guerre en tant «cause commune» permet d'expliquer, bien plus sûrement que la représentation politique, le maintien de la domination de Buenos Aires sur son ancienne juridiction. Ce lien ne survivrait pas sans la guerre, sans les échanges et les relations de réciprocité qui se créent entre la capitale et les cités de l'Intérieur, sans la visée commune de vaincre l'ennemi royaliste. Fondement du lien politique, la guerre dote le pouvoir central et ses acteurs d'une nouvelle légitimité, influe sur le cours de la révolution et notamment sur le choix délibéré du centralisme à partir de 1816 .

Dans le même temps, le conflit provoque une mobilisation des provinces qui modifie l'organisation interne de celles-ci, rapprochant et intégrant dans un nou-

47 Oficios de Abraham González al gobernador de Buenos Aires, 29 de agosto y 7 de septiembre de 1821, AGN, Gobierno de Tucumán, Sala X, legajo 5-10-5.

48 Oficio del cabildo de Tucumán al de Buenos Aires, 11 de septiembre de 1821, AGN, Gobierno de Tucumán, Sala X, legajo 5-10-5. 
veau type d'espace politique les cités et leurs campagnes environnantes (comarcas). Face à des responsabilités accrues, les provinces se dotent des instruments adéquats pour y parvenir, notamment en termes financiers. Leur faculté de se gouverner elles-mêmes augmente donc en même temps que se multiplient les relations entre elles et que se forge la conscience d'une solidarité commune. Peu à peu, elles se détachent de Buenos Aires qui ne peut plus assumer la conduite de la guerre, jusqu'à proclamer leurs indépendances respectives en 1820. Cela ne les empêche pas, bien au contraire, de maintenir entre elles un lien politique qui leur permet de poursuivre le combat contre l'ennemi commun.

Cependant, à côté de cette «guerre d'indépendance» existe un autre type de conflit, qui met d'abord aux prises les deux options du camp patriote («fédéral» et centraliste), puis plusieurs provinces entre elles. Les guerres du Littoral, et celles qui se multiplient après 1820, appartiennent à cette seconde catégorie que les acteurs nomment «guerres civiles» ${ }^{49}$. Moins violentes et moins coûteuses en hommes, elles s'inscrivent en quelque sorte dans le jeu des relations entre entités à prétention souveraine et, par là même, participent pleinement de la construction du lien politique. Alors que la guerre contre les royalistes définit une communauté de type nouveau, ces guerres inter-provinciales, et les traités auxquels elles donnent lieu, servent de fondement à l'organisation politique de ladite communauté, jouant par là même le rôle de textes constitutionnels. De fait, jusqu'à la Constitution de 1853, ces pactes inter-provinciaux (notamment celui de 1831) sont les seuls textes juridiques qui donnent une consistance à l'idée d'une union des provinces dans l'ancienne vice-royauté du Río de la Plata.

\section{BIBLIOGRAFÍA}

Acevedo, Eduardo, José Artigas, jefe de los orientales y protector de los Pueblos Libres. Alegato histórico, 2 vols., Montevideo, El Siglo Ilustrado, 1909.

Azcuy Ameghino, Eduardo, Artigas en la historia argentina, Buenos Aires, Corregidor, 1986.

Bazán, Armando Raúl, Historia del Noroeste argentino, Buenos Aires, Plus Ultra, 1986.

Bidondo, Emilio E., La guerra de la independencia en el norte argentino, Buenos Aires, Eudeba, 1976.

49 Nous suivons Clément Thibaud qui les qualifie plutôt de guerres «civiques», en tant qu'elles opposent des cités entre elles (Thibaud, 2003). 
Bidondo, Emilio E., La expedición de auxilio a las provincias interiores (1810-1812), Buenos Aires, Círculo Militar, 1987.

Bidondo, Emilio E., «Los ejercitos de la revolución: 25 de mayo de 1810-9 de julio de 1816», Investigaciones y Ensayos, 37 (Buenos Aires, 1988): 337-375.

Bernand, Carmen, «La poblacion negra de Buenos Aires (1777-1862)», Monica Quijada, Carmen Bernand, Arnd Schneider, Homogeneidad y Nación. Con un estudio de caso: Argentina, siglos XIX y XX, Madrid, CSIC, 2000: 93-140.

Caillet-Bois, Ricardo, «Artigas, defensor de la Revolución de Mayo y símbolo de la hermandad rioplatense», Boletín de la Academia Nacional de la Historia, 46 (Buenos Aires, 1973): 111-124.

Carranza, Adolfo P., Archivo General de la República Argentina, 14 vols., Buenos Aires, G. Kraft, 1894-1898.

Chiaramonte, José Carlos, «¿Provincias o Estados? Los orígenes del federalismo rioplatense», François-Xavier Guerra (ed.), Las revoluciones hispánicas: independencias americanas y liberalismo español, Madrid, Complutense, 1995: 167-205.

Chiaramonte, José Carlos, «Formas de identidad en el Río de la Plata luego de 1810», Boletín del Instituto de Historia Argentina e Americana «Dr. Emilio Ravignani», III/1 (Buenos Aires, 1989): 71-91.

Cornejo, Atilio, Historia de Güemes, Buenos Aires, Espasa-Calpe, 1946.

Demélas-Bohy, Marie-Danielle, Nacimiento de la guerra de guerrilla: el diario de José Santos Vargas (1810-1825), La Paz, Plural-Lima, IFEA, 2007.

Demicheli, Alberto, Formacion nacional rioplatense. Artigas y su obra jurídico-politica, 3 vols., Montevideo, Barrero y Ramos, 1955.

Díaz Bellido, Ana María, L'invention de l'indépendance du Paraguay: le discours officiel, vecteur d'un imaginaire, Thèse de doctorat, Université de Poitiers, 1999.

Figueroa Güemes, Martín G., La gloria de Güemes, Buenos Aires, Eudeba, 1971.

Fitte, Ernesto J., «El fusilamiento de los Carrera y las publicaciones de la época», Investigaciones y Ensayos, 20 (Buenos Aires, 1976): 177-203.

Frías, Bernardo, Historia del General Martín Güemes y de la provincia de Salta, 6 vols., Buenos Aires, Depalma, 1971-1973 (1. . ed. Salta, 1902).

Freyre, Ricardo J., Tucumán en 1810. Noticia histórica y documentos inéditos, Tucumán, 1909.

Gallo, Klaus, Las invasiones inglesas, Buenos Aires, Eudeba, 2004.

González Bernaldo, Pilar, «Produccion de una nueva legitimidad: ejército y sociedades patrióticas en Buenos Aires entre 1810 y 1813», Cahiers des Amériques Latines, 10 (Paris, 1990): 177-195. 
Guerra, François-Xavier, Modernidad e independencias. Ensayo sobre las revoluciones hispánicas, Madrid, Mapfre, 1992.

Halperin Donghi, Tulio, Revolución y guerra. Formación de una elite dirigente en la Argentina criolla, Buenos Aires, Siglo XXI, 1972.

Halperín Donghi, Tulio, «Militarización revolucionaria en Buenos Aires, 1806-1815», Tulio Halperín Donghi, El ocaso del orden colonial en Hispanoamérica, Buenos Aires, Sudamericana, 1978: 121-158.

Halperín Donghi, Tulio, Guerra y finanzas en los orígenes del Estado argentino (1791-1850), Buenos Aires, Editorial de Belgrano, 1982.

Hébrard, Véronique, Le Venezuela indépendant. Une nation par le discours, 1810-1830, Paris, L'Harmattan, 1996.

Heredia, Edmundo E., «Algunos aspectos de la reconquista del Río de la Plata durante el regimen constitucional español», Anuario de Estudios Americanos, 34 (Séville, 1977): 49-63.

Hobsbawm, Éric, Nations et nationalismes depuis 1780, Paris, Gallimard, 1992.

García Belsunce, Cesar A., «La Revolución de 1810 en Córdoba. Gobierno de Pueyrredón», Academia Nacional de la Historia, Tercer Congreso Internacional de Historia de América, Buenos Aires, 1961, vol. 6: 153-175.

González, Ariosto D., Las primeras fórmulas constitucionales en los países del Plata (1810-1814), Montevideo, Barrero y Ramos, 1955.

Güemes, Luis, Güemes documentado, 12 vols., Buenos Aires, Plus Ultra, 1977-1990.

Gutiérrez Ardila, Daniel, Un Nouveau Royaume. Géographie politique, pactisme et diplomatie durant l'interrègne en Nouvelle Grenade (1808-1816), Thèse de doctorat, Université de Paris I, octobre 2008.

McFarlane, Anthony, «Guerras e independencias en las Américas», María Teresa Calderón, Clément Thibaud ed., Revoluciones en el Mundo Atlántico, Bogotá, Externado de Colombia CEHIS-Taurus, 2006: 171-188.

Meisel, Seith, «El servicio militar y la construcción del Estado en Córdoba, Argentina, 1810-1840», Marta Terán, José Antonio Serrano (ed.), Las guerras de independencia en la América Española, Zamora, Instituto Nacional de Antropología e Historia-Universidad Michoacana de San Nicolás de Hidalgo, 2002: 439-451.

Ortiz Escamilla, Juan, Guerra y gobierno. Los pueblos y la independencia de México, Séville, Universidad Internacional de Andalucía-Universidad de Sevilla-Colegio de México-Instituto Mora, 1997.

Pastor, Reynaldo, «Acción de Güemes en el norte argentino», Academia Nacional de la Historia, Primer Congreso de Historia argentina y regional (Tucumán, 1971), Buenos Aires, ANH, 1973: 67-77. 
Petit Muñoz, Eugenio, Artigas: federalismo y soberanía, Montevideo, Universidad de la República, 1988.

Portillo Valdés, José María, Revolución de nación. Orígenes de la cultura constitucional en España, 1780-1812, Madrid, CEPC, 2000.

Puente Candamo, José A. de la, «Buenos Aires y el Peru en el tiempo de Abascal (1806-1816)», in Academia Nacional de la Historia, Sexto Congreso Internacional de Historia de América, Buenos Aires, 1980, vol. 5: 135-143.

Ramos Pérez, Demetrio, San Martín, el Libertador del Sur, Madrid, Anaya, 1988.

Reyes Abadie, Washington, Historia uruguaya, vol. II: 1810-1820: Artigas y el federalismo en el Río de la Plata, Montevideo, Ediciones de la Banda Oriental, 1975.

Salas, Reubén D., «Los proyectos monárquicos en el proceso de la independencia argentina (1810-1820)», Ibero-Amerikanisches Archiv, XV/2 (Berlin, 1989): 193-231.

Sampay, Arturo E., Las constituciones de la Argentina, Buenos Aires, Eudeba, 1975.

Segreti, Carlos, «La misión Allende-Villegas para poner paz en el Litoral», Trabajos y Comunicaciones, 18 (La Plata, 1968): 225-242.

Segreti Carlos, «La independencia de Córdoba en 1815», Academia Nacional de la Historia, Cuarto Congreso Internacional de Historia de América, Buenos Aires, 1966, vol. 2: 443-483.

Segreti, Carlos, La máscara de la monarquía (1808-1819), Córdoba, Centro de Estudios Históricos, 1994.

Thibaud, Clément, Repúblicas en armas. Los ejércitos bolivarianos en la guerra de independencia en Colombia y Venezuela, Bogotá, IFEA-Planeta, 2003.

Tio Vallejo, Gabriela, «Revolución y guerra en Tucumán, los procesos electorales y la militarización de la política», Marta Terán et José Antonio Serrano (ed.), Las guerras de independencia en la América Española, Zamora, Instituto Nacional de Antropología e Historia-Universidad Michoacana de San Nicolás de Hidalgo, 2002: 355-388.

Verdo, Geneviève, L'indépendance argentine entre cités et nation, Paris, Publications de la Sorbonne, 2006.

Verdo, Geneviève, «El dilema constitucional en las Provincias Unidas del Río de la Plata (1810-1819)», Historia Contemporánea, 31 (Bilbao, 2006): 513-536.

Vogel, Hans, «New Citizens for a New Nation: Naturalization in Early Independent Argentina», Hispanic American Historical Review, LXXI/1 (Durham, 1991): 107-131. 
THE CONSTITUENT WAR: RÍO DE LA PLATA, 1810-1821

In the perspective opened by the recent historiography, this article explores the political repercussions of the conflict in the Rio de la Plata. The war against the royalists acquires the character of a common identity, that explains how Buenos Aires manages to keep political control on the former virreinato. After 1820, the fight against the royalists is the main driving force of the union of independent provinces. At the same time, other types of conflicts are emerging, making up a particular expression of the political link under construction.

Key words: War, Independence, Río de la Plata, Political construction, Enemy, Comunities, Armies. 